\title{
Scientific Results from the FIRST Instrument Deployment to Cerro Toco, Chile and from the Flight of the INFLAME Instrument
}

\author{
M. G. Mlynczak, D. G. Johnson, and R. P. Cageao \\ NASA Langley Research Center, Hampton, VA, 23681 \\ m.g.mlynczak@nasa.gov
}

\begin{abstract}
Results from the FIRST and INFLAME infrared Fourier Transform Spectrometers are presented. These are comprehensive measurements of the far-IR spectrum (FIRST) and the net infrared fluxes within the atmosphere (INFLAME).

OCIS Codes: (010.0280) Remote Sensing and Sensors; 010.5620) Radiative Transfer
\end{abstract}

\section{Introduction}

For the past decade, NASA Langley Research Center has been developing and deploying unique instruments designed to enable a better understanding of the flow of infrared radiation with the Earth's atmosphere. These include the Far-Infrared Spectroscopy of the Troposphere (FIRST) instrument, which is a Fourier Transform Spectrometer (FTS) designed to accurately measure the far-infrared (far-IR) spectrum between 15 and $100 \mu \mathrm{m}(667$ to 100 wavenumbers.) This portion of the spectrum is not directly measured by any sensor currently in orbit or presently manifested for flight. However, the far-IR radiation plays a critical role in Earth's energy balance. Globally, approximately half of the outgoing longwave radiation emitted by the Earth to space is in the far-IR. Considering only the atmosphere, $\sim 75 \%$ of the infrared emission from the atmosphere is in the far-IR. The main constituent that governs the far-IR atmospheric emission in clear skies is water vapor, the main greenhouse gas within the atmosphere. Cirrus clouds also play a significant role in modulating the far-infrared emission to space.

In addition, the In-Situ Net Flux within the Atmosphere of the Earth (INFLAME) instruments have also been developed. The INFLAME instruments are also Fourier Transform Spectrometers, one for the visible portion of the spectrum, one for the infrared. They are designed to operate from an aircraft, measuring the upwelling, downwelling, and net fluxes of infrared radiation within the atmosphere. From these measurements, made at different altitudes, the vertical profiles of radiative heating and cooling are derived. The INFLAME instruments directly measure the net fluxes of radiation and are unique sensors for probing infrared radiation within the atmosphere.

\section{The Far-Infrared Spectroscopy of the Troposphere (FIRST) Instrument}

The Far-Infrared Spectroscopy of the Troposphere (FIRST) Instrument was proposed to the NASA Earth Science Technology Office Instrument Incubator Program in 2001 and was selected for development late that year. The goal of the FIRST project was to develop and demonstrate the technology necessary to make measurements of the farinfrared and infrared spectrum in a single instrument on a single focal plane.

FIRST is a high-throughput $\left(0.47 \mathrm{~cm}^{2}\right.$ sr $)$ Fourier transform spectrometer with a germanium on polypropylene beamsplitter. The FIRST instrument is housed in a vacuum container and can be cooled to $\sim 180 \mathrm{~K}$ in order to simulate the space environment and the lowest temperatures likely to be attained on orbit. The instrument has a scene select mirror that allows for viewing of the atmosphere as well as the two blackbody ports for calibration.

FIRST was demonstrated on a high altitude ( 105,000 feet) balloon on June 7, 2005 from the NASA Columbia Scientific Balloon Facility in Fort Sumner, NM. The instrument operated nominally for approximately 6 hours and recorded several thousand spectra on 10 discrete detectors. The instrument succeeded in demonstrating the technology and ability to measure the infrared spectrum, including the far-infrared spectrum, from 200 to $5 \mu \mathrm{m}$ (50 to 2000 wavenumbers). The FIRST instrument flew again from Ft. Sumner in September 2006, in support of validation activities associated with the "A-Train" series of satellites. The instrument operated nominally again for 6 hours at "float" altitude near $30 \mathrm{~km}$.

The FIRST instrument has now transitioned from an "incubator" and "technology demonstration unit" to a fullfledged science instrument. Over the course of 2007 and 2008, a second blackbody was built and the scene select 
assembly was rotated so that the instrument could view the zenith direction and thus operate from the ground. The second blackbody operates at a different temperature, offering the standard 2-point calibration for the FTS.

The modifications to enable ground operation of FIRST was to allow participation in field experiments conducted by the Department of Energy Atmospheric System Research (ASR) program. Specifically, from July to November 2009, FIRST participated in the Radiative Heating in the Underexplored Bands Campaign - II (RHUBC-II). The campaign was conducted on the top of Cerro Toco, a mountain 17,600 feet above sea level in the Atacama Desert of Chile. FIRST was operated at this altitude along with 3 other FTS instruments to record the entire downwelling infrared spectrum of Earth's atmosphere from the sub-millimeter to the near-infrared at high spectral resolution. The purpose of RHUBC-II was to conduct a radiative closure experiment, in which measurements of infrared emission spectra would be compared with calculated spectra. The inputs for the calculations are provided from radiosonde data taken simultaneously with the infrared spectral measurements. The location (and altitude) of the experiment were chosen so as to be above most of the water vapor and thus avail the spectral development of the far-infrared to be observed down as low as 200 wavenumbers.

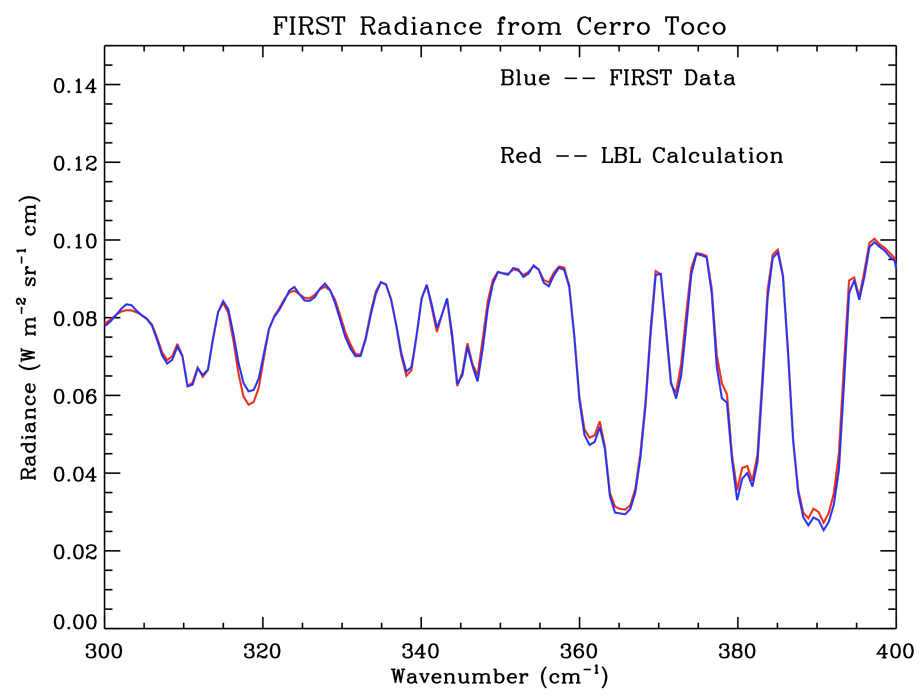

Fig. 1. FIRST Measured (blue) and computed (red) spectra between 300 and 400 wavenumbers at Cerro Toco, Chile in September 2009.

Shown in Figure 1 in the blue curve is an observed far-infrared spectrum recorded by the FIRST instrument between 300 and 400 wavenumbers at Cerro Toco. The red curve is a line-by-line calculation of the radiance based on water vapor and temperature profiles simultaneously recorded via radiosonde.

\section{The In-Situ Net Flux within with Atmosphere of the Earth (INFLAME) Instrument}

As important as the measurement of infrared spectra is at the top of the atmosphere and from the surface looking up, it is also crucially important to understand radiation fields within the atmosphere. The divergence of the net flux of radiation determines the rate at which the atmosphere heats or cools by radiative transfer. However, accurately measuring radiative heating rates within the atmosphere is perhaps the most difficult measurement to accomplish.

The fundamental equations solved in every atmospheric model include the momentum and continuity equations and the thermodynamic equation or equivalently, the first law of thermodynamics. The main terms in the first law that must be determined are the rates of atmospheric heating and cooling due to absorption of solar radiation and emission of infrared radiation. The radiative heating rate $d T / d t$ is determined from the expression:

$$
\frac{d T}{d t}=\frac{-1}{\rho C_{p}} \frac{d F_{n e t}}{d z}
$$


where $\rho$ is atmospheric density, $c_{p}$ is the heat capacity at constant pressure, and $F_{n e t}$ is the net radiative flux at altitude $z$. The net flux is simply the difference between the energy flowing upward and downward through an aperture of unit area, or $F_{n e t}=F_{\uparrow}-F_{\downarrow}$. The change in net flux with altitude is the net flux divergence, $d F_{n e t} / d z$, and is proportional to the rate $d T / d t$ at which radiation heats or cools the atmosphere. The net fluxes are usually separately determined for the visible (solar) and the infrared (thermal) parts of the spectrum.

The In-situ Net Flux within the Atmosphere of the Earth (INFLAME) project was also selected for development under the NASA Instrument Incubator Program. The goal is to measure the shortwave and longwave net flux with sufficient stability to derive tropospheric heating rates in $1 \mathrm{~km}$ layers that are accurate to within $10 \%$. This requires measuring the net flux with a stability of $0.2 \%$ per $\mathrm{km}$ and $0.8 \%$ per $\mathrm{km}$ in the shortwave and longwave spectral regions, respectively. It is important to note that while measuring the net flux divergence requires that the instrument response be very stable, it does not require a similar level of absolute accuracy in measuring the net flux. If the calibration errors are stable and independent of altitude then the relative uncertainty in the net flux divergence will be no greater than the relative uncertainty in the net flux measurement.

The INFLAME instruments measure the net flux by using a low-resolution Fourier transform spectrometer (FTS) to observe the upward and downward flux simultaneously using the two inputs of the same instrument. The two complementary outputs of the FTS can be transformed to produce spectra proportional to the difference between the two inputs.

The net flux is measured directly in the atmosphere by mounting the instruments in the wing tip tanks of a Lear Jet. The aircraft slowly ascends from near the surface to approximately 40,000 feet in altitude, recording the profile of net flux. As indicated by Equation 1, the derivative of the vertical profile of net flux gives the rate at which the atmosphere is heated. Shown in Figure 2 is the spectral infrared radiative cooling rate $\left(\mathrm{mK} \mathrm{day}^{-1} \mathrm{~cm}\right)$ measured by INFLAME. The flight took place over 2.5 hours just off the Virginia coastline in January 2010. Figure 2 is the first measurement ever of the spectral infrared radiative cooling rate in the atmosphere.

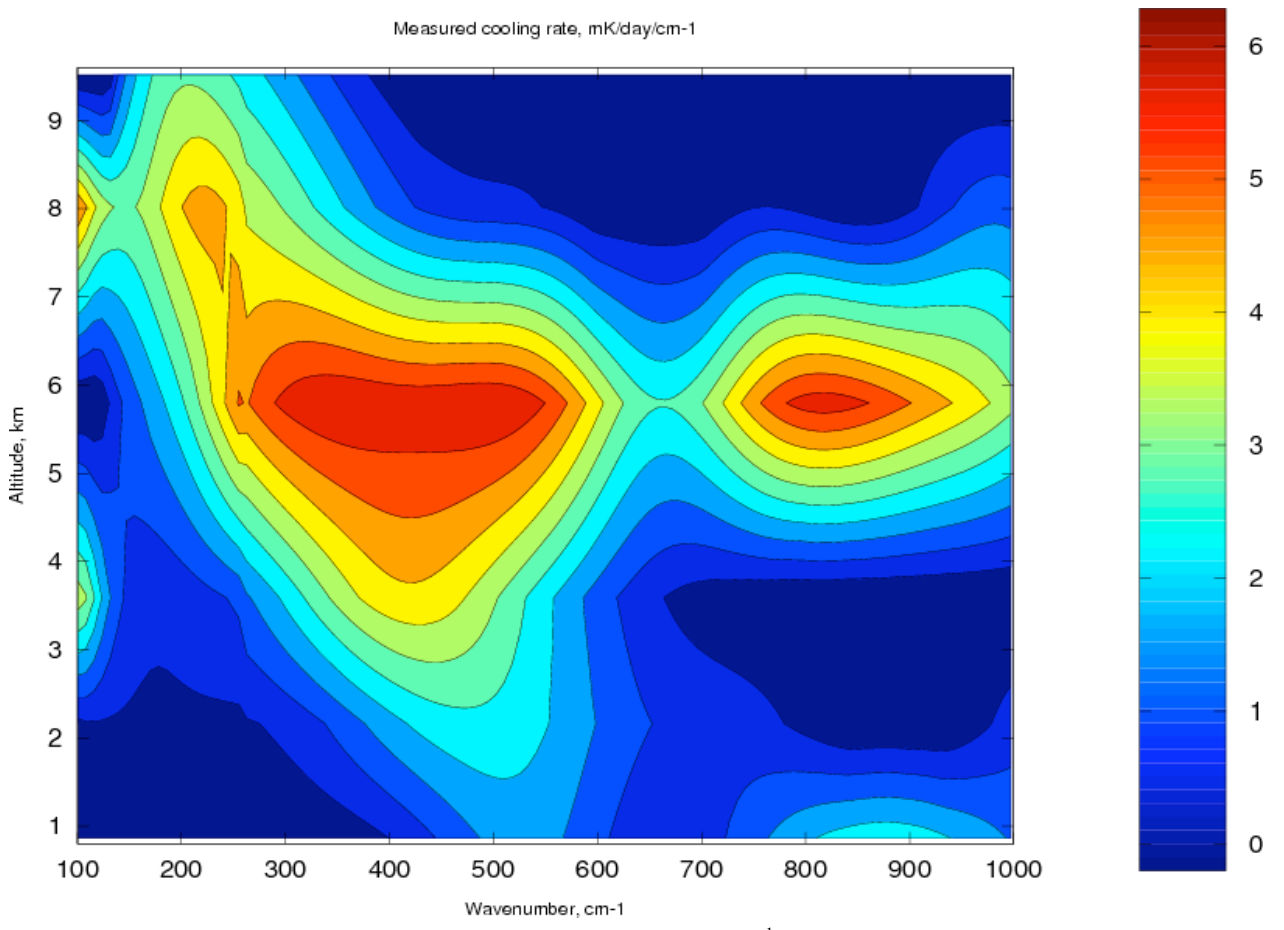

Fig. 2. Spectral radiative cooling rate, $\mathrm{mK} / \mathrm{day} / \mathrm{cm}^{-1}$, measured 5 January 2010

\section{Summary}

Two unique instruments, FIRST and INFLAME, have been developed and demonstrated. These provide new looks at the flow of infrared radiation at the top, bottom, and within the atmosphere.

We would like to acknowledge the support from the NASA Earth Science Technology Office and the many academic and industry partners who have worked with us over the years. 